\title{
Evaluation of hemodynamic goal-directed therapy to reduce the incidence of bone cement implantation syndrome in patients undergoing cemented hip arthroplasty - a randomized parallel-arm trial
}

\author{
Kai B. Kaufmann ${ }^{1,3^{*}}$ (D) Wolfgang Baar ${ }^{1,3}$, Judith Rexer ${ }^{1,3}$, Thomas Loeffler ${ }^{1,3}$, Sebastian Heinrich ${ }^{1,3}$,
} Lukas Konstantinidis ${ }^{2,3}$, Hartmut Buerkle ${ }^{1,3}$ and Ulrich Goebel ${ }^{1,3^{*}}$

\begin{abstract}
Background: The bone cement implantation syndrome (BCIS) is a frequent and potentially disastrous intraoperative complication in patients undergoing cemented hip arthroplasty. Several risk factors have been identified, however randomized controlled trials to reduce the incidence of BCIS are still pending. We hypothesized that goal-directed hemodynamic therapy guided by esophageal Doppler monitoring (EDM) may reduce the incidence of BCIS in a randomized, controlled parallel-arm trial.

Methods: After approval of the local ethics committee, 90 patients scheduled for cemented hip arthroplasty at the Medical Center - University of Freiburg were randomly assigned to either standard hemodynamic management or goal-directed therapy (GDT) guided by an esophageal Doppler monitoring-based algorithm. The primary endpoint was the incidence of overall BCIS including grade 1-3 after cementation of the femoral stem. Secondary endpoints included cardiac function, length of hospital stay and postoperative complications.

Results: Ninety patients were finally analyzed. With regards to the primary endpoint, the overall incidence of BCIS showed no difference between the GDT and control group. Compared to the control group, patients of the GDT group showed a higher cardiac index before and after bone cement implantation (2.7 vs. $2.2\left[\right.$ lø $\left.\mathrm{min}^{-1} \bullet \mathrm{m}^{-2}\right] ; 2.8$ vs. 2.4 $\left[l \bullet \mathrm{min}^{-1} \bullet \mathrm{m}^{-2}\right.$; $P=0.003, P=0.042$ ), whereas intraoperative amount of fluids and mean arterial pressure did not differ.

Conclusions: The implementation of a specific hemodynamic goal-directed therapy did not reduce the overall incidence of BCIS in patients undergoing cemented hip arthroplasty.

Trial registration: This randomized clinical two-arm parallel study was approved by the local Ethics Committee, Freiburg, Germany [EK 160/15, PI: U. Goebel] and registered in the German Clinical Trials Register (DRKS No. 00008778, 16th of June, 2015).
\end{abstract}

Keywords: Hip arthroplasty, Postoperative complications, Hemodynamics

\footnotetext{
* Correspondence: Kai.Kaufmann@uniklinik-freiburg.de;

Ulrich.Goebel@uniklinik-freiburg.de

${ }^{1}$ Department of Anaesthesiology and Critical Care, Medical Center -

University of Freiburg, Hugstetter Strasse 55, 79106 Freiburg, Germany

Full list of author information is available at the end of the article
}

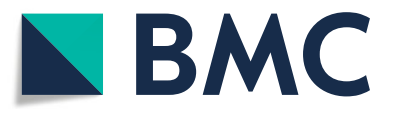

(c) The Author(s). 2018 Open Access This article is distributed under the terms of the Creative Commons Attribution 4.0 International License (http://creativecommons.org/licenses/by/4.0/), which permits unrestricted use, distribution, and

reproduction in any medium, provided you give appropriate credit to the original author(s) and the source, provide a link to the Creative Commons license, and indicate if changes were made. The Creative Commons Public Domain Dedication waiver (http://creativecommons.org/publicdomain/zero/1.0/) applies to the data made available in this article, unless otherwise stated. 


\section{Background}

The number of hemiarthroplasties to treat femoral neck fractures or total hip replacement surgery due to osteoarthritis is increasing steadily while patients' age and severe comorbidities increase concomitantly $[1,2]$. For several reasons orthopedic surgeons prefer cemented hip arthroplasty mainly for older patients reducing the rate of re-operation due to aseptic loosening [3]. Although safety guidelines for the reduction of bone cement implantation syndrome (BCIS) have been published recently [4], BCIS remains a frequent intraoperative complication with an overall incidence of up to $28 \%$ [5]. Up to date, the pathophysiology is not fully understood [5]. The main clinical problem is an acute right ventricular insufficiency due to pulmonary embolism caused by fat tissue and bone cement [6-8]. Donaldson and colleagues established a severity classification of BCIS according to oxygen desaturation and hypotension (Table 1) [6]. Patients with BCIS grade 2 and 3 showed a 16-fold increase in 30 day postoperative mortality compared to those with BCIS grade $1[5,9]$. Several independent predictors for severe BCIS have been identified: ASA grade 3-4, chronic obstructive pulmonary disease and preoperative medication with diuretics or warfarin [5].

A variety of studies with promising results have been conducted on a goal-directed hemodynamic therapy for patients scheduled for total hip replacement or repair of femoral neck fractures [10-12]. According to these studies esophageal Doppler monitor-guided (EDM) goal-directed therapy (GDT) shortened time to being medically fit for discharge and resulted in a significantly reduced length of hospital stay $[10,12]$. However, these studies did not focus on the incidence of BCIS. The Enhanced Recovery after Surgery (ERAS ${ }^{\circ}$ ) Society developed an evidence-based algorithm for goal-directed hemodynamic management which includes monitoring of cardiac output and optimization of stroke volume [13, 14]. Different hemodynamic monitoring methods (e.g., pulse contour method, pulmonary artery catheter, transesophageal echocardiography) have been used to guide individualized intraoperative management [15].
However, compared to the esophageal Doppler technique most of these methods are invasive, bearing an increased risk for complications [16-18]. Whereas the accuracy of EDM was validated in various studies [19]. The effect of EDM GDT on the incidence of BCIS during cemented hip arthroplasty has not been investigated.

The hypothesis of this study was that optimizing hemodynamic parameters by EDM guided GDT before cement implantation would reduce the incidence of BCIS.

\section{Methods}

This randomized clinical two-arm parallel study was approved by the local Ethics Committee, Freiburg, Germany [EK 160/15, PI: U. Goebel] and registered in the German Clinical Trials Register (DRKS No. 00008778, 16th of June, 2015). The study was conducted at the Department of Anesthesiology and Intensive Care and the Department of Orthopedic Surgery, Medical Center - University of Freiburg, Faculty of Medicine, University of Freiburg, Germany.

Patients admitted to the Department of Orthopedic Surgery at the Medical Center University of Freiburg between September 2015 and October 2016 were eligible for this study (Fig. 1). Inclusion criteria were cemented hip arthroplasty under general anesthesia, age $>18$ years and written informed consent. Exclusion criteria were body-mass-index $(\mathrm{BMI})>50$, esophageal- or gastric pathologies and pregnancy.

\section{Anesthetic protocol}

All patients enrolled in this study received a femoral nerve block in single shot technique before general anesthesia was started. This procedure was ultrasound-guided (in-plane approach) and conducted via a $22 \mathrm{G}$ needle (Pajunk, Geisingen, Germany). In total, a single dose of $20 \mathrm{ml}$ ropivacaine $0.2 \%$ was administered. Patients' radial artery was cannulated for continuous blood pressure and intermittent blood gas analysis. In patients with ASA classification $\geq 3$ a central venous line via the internal jugular vein was established. Anesthesia was induced with

Table 1 Definition of Bone cement implantation syndrome (BCIS) [6] and primary endpoint

\begin{tabular}{|c|c|c|c|}
\hline BCIS grade 1 & \multicolumn{3}{|c|}{ Moderate hypoxia $\left(\mathrm{SpO}_{2}<94 \%\right)$ or hypotension (fall in systolic blood pressure $(\mathrm{SBP})>20 \%$ ) } \\
\hline $\mathrm{BCIS}$ grade 2 & \multicolumn{3}{|c|}{ Severe hypoxia $\left(\mathrm{SpO}_{2}<88 \%\right.$ ) or hypotension (fall in SBP $>40 \%$ ) or unexpected loss of consciousness } \\
\hline BCIS grade 3 & \multicolumn{3}{|c|}{ Cardiovascular collapse requiring CPR } \\
\hline BCIS grade & $\begin{array}{l}\text { GDT } \\
(n=45)\end{array}$ & $\begin{array}{l}\text { Control } \\
(n=45)\end{array}$ & PValue \\
\hline 1 & $17(38)$ & $14(31)$ & 0.51 \\
\hline 2 & $0(0)$ & $7(16)$ & 0.006 \\
\hline 3 & 0 & 0 & \\
\hline Total & $17(38)$ & $21(47)$ & 0.39 \\
\hline
\end{tabular}

Data are presented as number of patients and percentage within the group. Results with a $P$ Value $<0.05$ are considered significant and are written in italic type GDT goal-directed therapy 


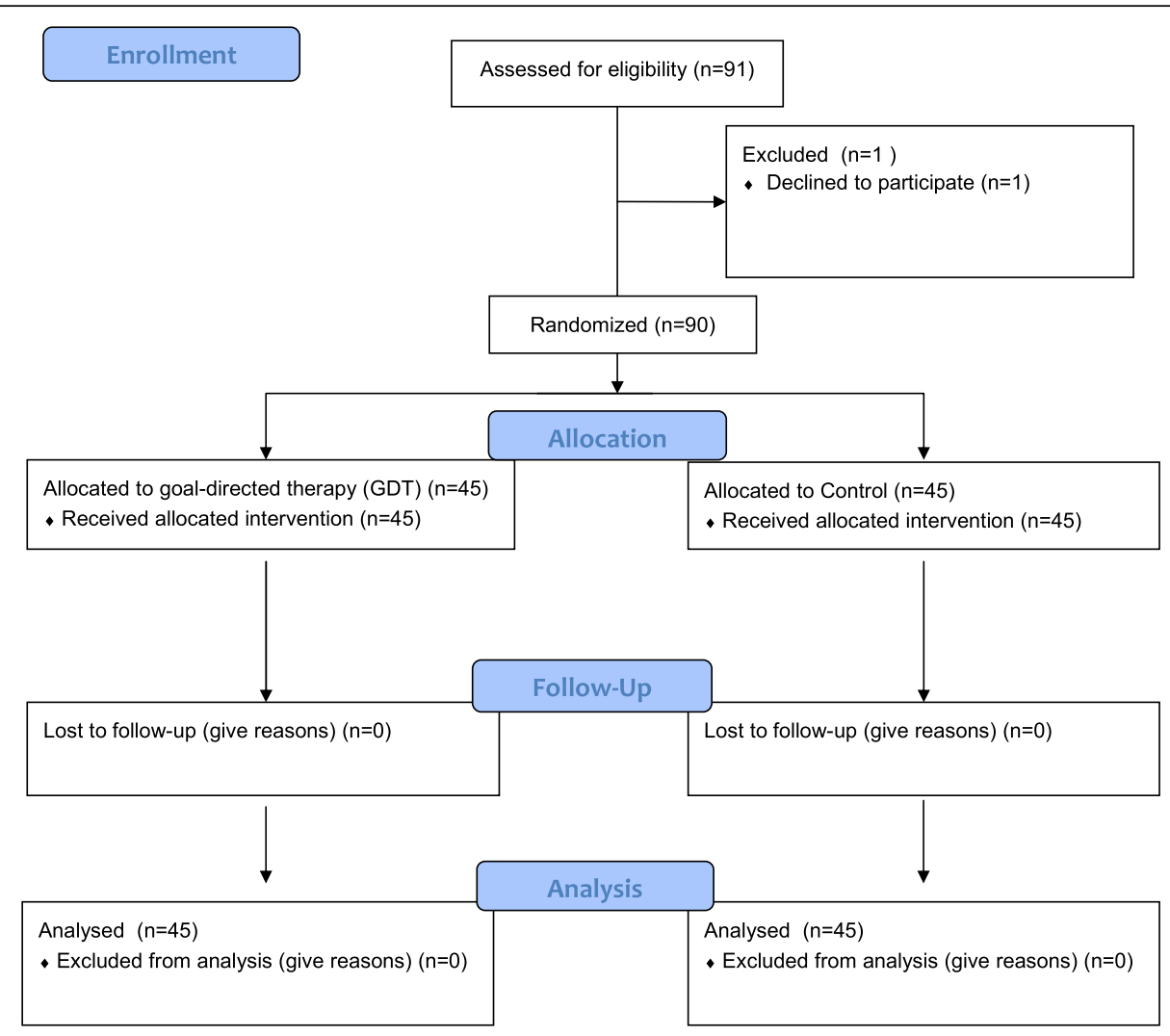

Fig. 1 CONSORT Statement

intravenous sufentanil $0.5 \mu \mathrm{g} \cdot \mathrm{kg}^{-1}$ and target-controlled infusion of propofol at plasma concentrations of 2.5-4 $\mu \mathrm{g} \cdot \mathrm{ml}^{-1}$ (Propofol 1\% MCT and Injectomat TIVA Agilia, Fresenius-Kabi GmbH, Bad Homburg, Germany). If patients had a history of postoperative nausea and vomiting propofol was used to maintain general anesthesia. Otherwise desflurane was used at a minimal alveolar concentration (MAC) of 0.9. To facilitate endotracheal intubation an intravenous bolus of cis-atracurium $\left(0.1 \mathrm{mg} \cdot \mathrm{kg} \mathrm{BW}^{-1}\right)$ was administered. During surgery, the grade of muscle relaxation was monitored using the quantitative and qualitative train-of-four technique. All patients were successfully extubated in the operating room and then transferred to the intensive care unit afterwards. The medical and nursing staff taking care of the patients on the ward were blinded to group assignment.

\section{Study design}

Based on a computer-generated list, patients were randomly allocated (using the sequentially numbered, opaque, sealed envelope technique) to one of the following two groups after induction of general anesthesia: (A) goal-directed therapy (GDT) group based on an EDM-guided algorithm (Fig. 2a); (B) control group with conventional fluid and hemodynamic management along standard operating procedures of the department of orthopedic anesthesia (Fig. 2b). The allocation ratio was 1:1. Written informed consent was obtained from all patients prior to enrolling them in the study. The nature of the study did not allow complete blinding of anesthesiologists. The anesthesiologist in charge treating the patient in the operating room had to stick either to the ERAS algorithm or to the control group management, so blinding in the operating room was not possible.

In both groups a Doppler probe was placed in the mid esophagus immediately after induction of anesthesia monitored by aortic visual and auditory signals (Deltex Medical ${ }^{\mathrm{Tm}}$, Chichester, United Kingdom). In patients of the control group, EDM (displaying stroke volume and cardiac index) was covered and made invisible to the treating anesthesiologist. In both groups intraoperative maintenance rate of crystalloid fluids was set to $3 \mathrm{ml} \bullet \mathrm{kg}^{-1} \bullet \mathrm{h}^{-1}$. EDM-derived hemodynamic data were analyzed at four time periods: before start of surgery, before bone cement insertion, after bone cement implantation into the femur and at the end of surgery. All EDM variables, including cardiac index, stroke volume, stroke distance, flow time, mean acceleration, peak velocity, heart rate and systemic arterial pressure were recorded every $5 \mathrm{~s}$.

Patients randomized to the GDT group were managed according to the ERAS $^{\circ}$ algorithm utilizing EDM 


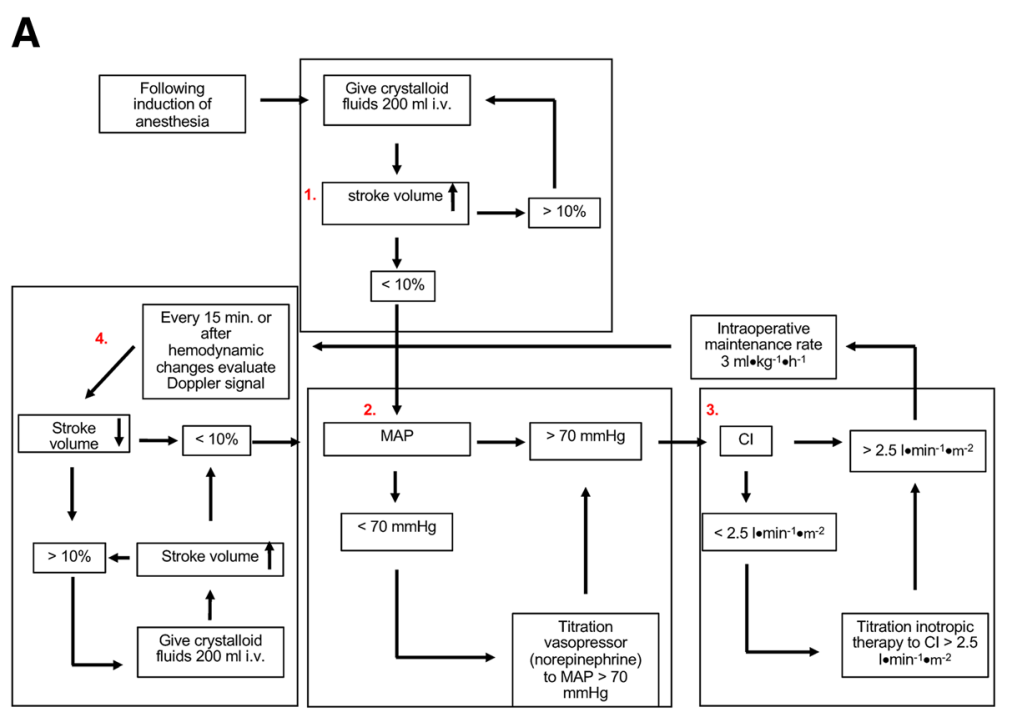

\section{B}

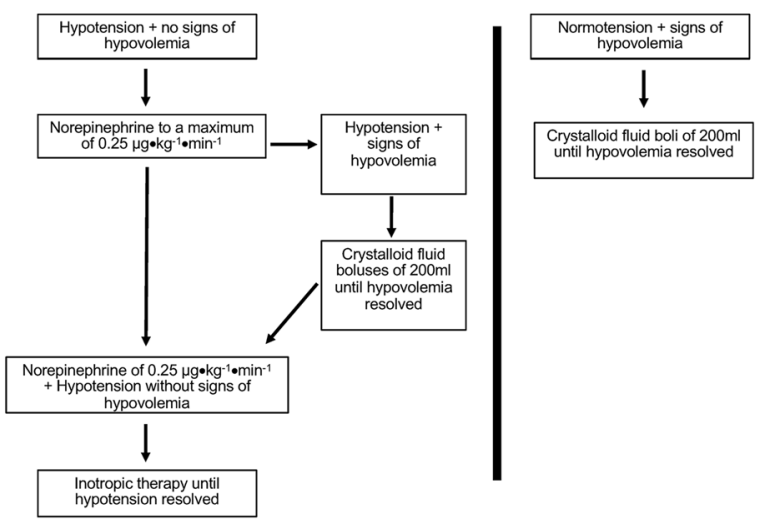

Fig. 2 (a) Goal-directed hemodynamic therapy (GDT) (b) Standard hemodynamic algorithm. (a) Goal-directed hemodynamic algorithm to guide intraoperative volume, vasopressor and inotropic therapy in the Goal-directed therapy group adopted from ERAS [13]. (b) Hemodynamic algorithm to guide intraoperative volume, vasopressor and inotropic therapy in the control group according to the standard operating procedure of the department of orthopedic anesthesia

variables (i.e., MAP, stroke volume and cardiac index) (Fig. 2a) Following induction of anesthesia a bolus of $200 \mathrm{ml}$ of crystalloid fluid was administered. If stroke volume increased by $\geq 10 \%$, additional boli of $200 \mathrm{ml}$ were given until the increase in stroke volume was less than $10 \%$. As long as MAP was less than $70 \mathrm{mmHg}$ and/ or cardiac index (CI) less than $2.51 \bullet \mathrm{min}^{-1} \bullet \mathrm{m}^{-2}$ despite an increase in stroke volume of less than $10 \%$ following fluid challenge, low dose norepinephrine by continuous infusion and/or ephedrine were administered, respectively. Fluid responsiveness and hemodynamic variables were re-assessed at least every $15 \mathrm{~min}$ and more frequently in case of hemodynamic instability or bone cement insertion into the femur (Fig. 2a).

Patients allocated to the control group were hemodynamically managed as follows. Isolated hypotension (defined as 20\% decrease in mean arterial blood pressure (MAP) below baseline or less than $60 \mathrm{mmHg}$ ) was treated by a continuous infusion of norepinephrine up to a maximum rate of $0.25 \mu \mathrm{g} \bullet \mathrm{kg}^{-1} \bullet \mathrm{min}^{-1}$. If hypotension persisted, inotropes were administered until MAP was $\geq 60 \mathrm{mmHg}$. In the control group the type of inotropic therapy was not predefined according to a study protocol. Therefore, dobutamine, epinephrine, and ephedrine were used according to individual preferences. If hypotension was accompanied by signs of hypovolemia (defined as urine output less than $0.5 \mathrm{ml} \bullet \mathrm{kg}^{-1} \bullet \mathrm{h}^{-1}$ and/or an increase in heart rate of more than $20 \%$ above baseline) crystalloid fluid was administered until urine output and/or heart rate normalized. If hypotension persisted despite volume challenges, norepinephrine was administered at the above listed dosage (Fig. 2b). 


\section{Outcome measures}

The primary endpoint was the incidence of BCIS as defined by Donaldson and colleagues (Table 1) [6]. From the start of our study, including the a-priori power analysis for sample size calculation we focused on the incidence of BCIS applying a standardized severity classification to detect differences in hemodynamics after bone cement implantation between the GDT and control group. The aim was to have patients' hemodynamic parameters optimized to the maximum at the time of cement implantation. This included the following key points which were optimized before the implantation of bone cement into the femur: preload, cardiac performance and mean arterial pressure. Our intention was to show that due to the applied hemodynamic algorithm, patients in the GDT group showed significantly improved hemodynamic parameters compared to the control group before bone cement insertion into the femur. We hypothesized that this significant, preemptive hemodynamic optimization due to the EDM guided algorithm reduces the risk of BCIS. Mean arterial pressure and cardiac performance was recorded every $5 \mathrm{~s}$. As described by Olsen and colleagues only periods of hypotension and oxygen desaturation that occurred within 15 min after bone cement insertion into the femur were considered for the diagnosis of BCIS [5].

Secondary endpoints included acute kidney injury (as defined by the Acute Kidney Injury Network Criteria), cardiac morbidity (myocardial infarction as diagnosed by electrocardiogram and/or troponin $\mathrm{T}$ serum concentration; newly developed atrial fibrillation), neurological morbidity (stroke and delirium), wound healing disorder (drainage of pus from surgical wound) and pulmonary complications (respiratory infection, respiratory failure, pleural effusion, atelectasis, pneumothorax, bronchospasm, aspiration pneumonitis) [20]. Length of hospital stay was documented. Secondary endpoints were re-assessed within 30 days after surgery. All outcomes were documented by research personnel unaware of patients' group assignment.

\section{Statistical analysis}

The sample size calculation was based on a previously reported overall incidence of BCIS during cemented hip arthroplasty of approximately $28 \%$ [5]. Due to the clinical experience of our department of orthopedic anesthesia with a low incidence of overall BCIS of $5 \%$ for patients with cemented hip arthroplasty we calculated a sample size of 45 patients per group with a power of $80 \%$ and a two-sided significance level of $5 \%$. We hypothesized that this low incidence of BCIS was the result of a specific hemodynamic goal-directed therapy guided by EDM. We used the incidence of overall BCIS for sample size calculation, aware that this study is under-powered to draw conclusions on results about
BCIS grade 2 and 3 which are post hoc subgroup findings. Categorical data were analyzed using Fisher's exact test. Continuous variables were examined for normal distribution. If data were normally distributed, independent samples t-test for normally distributed variables was performed. In case of not normally distributed data, the Mann-Whitney U test was used. Statistical analysis was performed using IBM $^{\mathrm{rm}}$, (Armonk, United States) SPSS Statistics 22 software.

\section{Results}

The consort diagram is shown in Fig. 1. Ninety-one patients were assessed for eligibility between September 2015 and October 2016. One patient was excluded because formal informed consent was withdrawn. Ninety patients were randomly allocated to the goal-directed (GDT) or control group. Finally, 45 patients were analyzed in each group. No adverse events occurred due to EDM probe placement.

\section{Primary endpoint}

The overall incidence of BCIS did not differ significantly between the GDT and control group (Table 1). Post hoc subgroup analysis showed a difference for BCIS grade 2 . In the GDT group, none of the patients showed BCIS grade 2 whereas 7 patients $(16 \%)$ of the control group $\operatorname{did}(P=0.006)$.

\section{Secondary endpoints}

The incidences of postoperative wound healing disorder, neurological, pulmonary or cardiac complications were comparable between groups. One patient (2\%) of the GDT group compared to 6 patients (13\%) of the control group developed postoperative acute kidney injury $(P=0.049)$. With regards to the incidence of overall complications there was a trend of less complications in the GDT group (Table 2).

\section{Baseline patient characteristics and perioperative parameters}

Despite all other parameters, the number of patients with chronic kidney disease stage 3 diagnosed by a preoperative glomerular filtration ratio of 30 to $60 \mathrm{ml}$ differed significantly between both groups (Table 3 ).

Anesthesia and surgery time intervals, total blood loss, urinary excretion and administered crystalloids were comparable between the groups. With regards to the intraoperative catecholamine therapy the two groups showed significant differences. Patients of the GDT group had less norepinephrine but received inotropic medication more often (Table 4). The need for vasopressor therapy after surgery was comparable between both groups. In addition, length of hospital stay was also comparable between groups (Table 4). 
Table 2 Postoperative complications

\begin{tabular}{llll}
\hline & $\begin{array}{l}\text { GDT } \\
(\mathrm{n}=45)\end{array}$ & $\begin{array}{l}\text { Control } \\
(n=45)\end{array}$ & P Value \\
\hline Acute kidney injury & $1(2)$ & $6(13)$ & 0.049 \\
Neurological & $3(7)$ & $2(4)$ & 0.65 \\
Wound healing disorder & $1(2)$ & $3(7)$ & 0.31 \\
Pulmonary & $7(16)$ & $7(16)$ & 1.0 \\
Cardiac & 0 & $1(2)$ & 0.32 \\
Total & $10(22)$ & $17(38)$ & 0.11 \\
\hline
\end{tabular}

Data are presented as number of patients and percentage within the group GDT goal-directed therapy. Results with a $P$ Value $<0.05$ are considered significant and are written in italic type

Table 3 Baseline patient characteristics

\begin{tabular}{|c|c|c|c|}
\hline & $\begin{array}{l}\text { GDT } \\
(n=45)\end{array}$ & $\begin{array}{l}\text { Control } \\
(n=45)\end{array}$ & $P$ Value \\
\hline Male/female & $33 \% / 66 \%$ & $44 \%$ / $56 \%$ & 0.28 \\
\hline Age & $79(74.5-83.5)$ & $79(72.5-83)$ & 0.61 \\
\hline Body mass index & $24.8(22.3-28.3)$ & 24.6 (21.4-26.8) & 0.44 \\
\hline ASA 1 & $0 \%$ & $0 \%$ & \\
\hline ASA 2 & $17(38)$ & $10(22)$ & 0.11 \\
\hline ASA 3 & $23(51)$ & $28(62)$ & 0.29 \\
\hline ASA 4 & $5(11)$ & $7(16)$ & 0.54 \\
\hline CAD & $5(11)$ & $10(22)$ & 0.16 \\
\hline Hypertension & $30(67)$ & $24(53)$ & 0.2 \\
\hline Liver disease & 0 & $1(2)$ & 0.32 \\
\hline $\mathrm{GFR}>90 \mathrm{ml}$ & $4(9)$ & $7(16)$ & 0.33 \\
\hline GFR $60-90 \mathrm{ml}$ & $11(24)$ & $14(31)$ & 0.48 \\
\hline GFR $30-60 \mathrm{ml}$ & $28(62)$ & $17(38)$ & 0.02 \\
\hline GFR $15-30 \mathrm{ml}$ & $2(4)$ & $7(16)$ & 0.08 \\
\hline Diabetes & $9(20)$ & $9(20)$ & 1.00 \\
\hline Beta receptor blocker & $14(31)$ & $19(42)$ & 0.27 \\
\hline Anticoagulation & $18(40)$ & $18(40)$ & 1.00 \\
\hline Acetylsalicyl acid & $9(20)$ & $12(27)$ & 0.46 \\
\hline ACE inhibitor & $13(29)$ & $11(24)$ & 0.63 \\
\hline ARBs & $8(18)$ & $12(27)$ & 0.31 \\
\hline Diuretics & $11(24)$ & $15(33)$ & 0.35 \\
\hline Nicotine dependence & $3(7)$ & $5(11)$ & 0.46 \\
\hline \multicolumn{4}{|l|}{ Indication for surgery: } \\
\hline Trauma & $20(44)$ & $19(42)$ & 0.83 \\
\hline Arthrosis & $8(36)$ & $8(36)$ & 1.00 \\
\hline Infectious & $6(13)$ & $7(16)$ & 0.76 \\
\hline Necrosis & $3(7)$ & $0(0)$ & 0.08 \\
\hline Osteolysis & $0(0)$ & $3(7)$ & 0.08 \\
\hline
\end{tabular}

Data are presented as number of patients and percentage within the group or median and interquartile range

ASA American Society of Anaesthesiologists, GDT goal-directed therapy, CAD coronary artery disease, $A C E$ inhibitor angiotensin-converting enzyme inhibitor $A R B s$ Angiotensin-receptor-II blockers, GFR glomerular filtration ratio. Results with a $P$ Value $<0.05$ are considered significant and are written in italic type
Intraoperative hemodynamic parameters

For all four time periods mean acceleration (MA), heart rate (HR), peak velocity (PV), systolic (BP sys) and mean arterial pressure (MAP) did not differ between groups. Patients of the GDT group showed an increased stroke distance and flow time prior to cement implantation. The cardiac index and stroke volume index before, after and at the end of surgery differed between the GDT and control group although this difference was not present at the beginning of surgery (Table 5).

\section{Discussion}

The main results of this study can be summarized as follows: First, EDM GDT did not reduce the rate of overall BCIS including grade $1-3$. Post hoc subgroup analysis showed a reduction of BCIS grade 2 in the GDT group. Second, due to EDM GDT, patients showed better cardiac performance with no differences in MAP by using more inotropes, less vasopressors but the same amount of fluids. Third, despite an increased number of patients with preoperative renal dysfunction in the GDT group according to the baseline characteristics, these patients showed a decreased incidence of postoperative acute kidney injury.

The overall incidence of BCIS in this study is higher than described in current literature [5]. The reason for this discrepancy could be the overall increased systolic blood pressure just before cement implantation which was obvious in both groups as a result of volume or catecholamine therapy. Communication with the surgical team at the moment of bone cement implantation is essential in the management of BCIS and is part of our standard operating procedure independent of study group assignment. This may explain the mentioned iatrogenic increase in systolic blood pressure immediately before bone cement implantation in this study. Thus, a decrease of systolic blood pressure to diagnose BCIS might become more obvious. Another reason for this discrepancy might be that Olsen and colleagues collected data from a chart review on systolic blood pressure every fifth minute for a period of at least $15 \mathrm{~min}$ after bone cement implantation. In this randomized trial, systolic blood pressure was recorded every five seconds continuously so that our approach is more sensitive.

Although BCIS is described as a multifactorial phenomenon the acute right ventricular dysfunction due to pulmonary embolism is a key feature $[6,21]$. Compared to the control group EDM in the GDT group provides insights into cardiac function; i.e. hemodynamic parameters. The aim was to have patients' hemodynamic parameters optimized to the maximum at the time of cement implantation. This included the following key points which were optimized before bone cement implantation: preload, cardiac performance and mean arterial pressure. We 
Table 4 Perioperative data

\begin{tabular}{|c|c|c|c|}
\hline & $\begin{array}{l}\text { GDT } \\
(n=45)\end{array}$ & $\begin{array}{l}\text { Control } \\
(n=45)\end{array}$ & $P$ Value \\
\hline Anesthesia (min) & $208 \pm 57$ & $231 \pm 66$ & 0.09 \\
\hline Surgery (min) & $117 \pm 42$ & $127 \pm 55$ & 0.34 \\
\hline Blood loss (ml) & $504 \pm 407$ & $410 \pm 354$ & 0.29 \\
\hline Urinary excretion $\left(\mathrm{m} \mid \bullet \mathrm{kg}^{-1} \bullet \mathrm{h}^{-1}\right)$ & $2.9 \pm 2.7$ & $3.3 \pm 2.2$ & 0.60 \\
\hline Crystalloids $\left(\mathrm{ml} \bullet \mathrm{kg}^{-1} \bullet \mathrm{h}^{-1}\right.$ ) & $13 \pm 5$ & $13 \pm 5$ & 0.42 \\
\hline Norepinephrine $\left(\mu \mathrm{g} \bullet \mathrm{kg}^{-1} \bullet \mathrm{min}^{-1}\right)$ & $0.03 \pm 0.03$ & $0.05 \pm 0.03$ & 0.05 \\
\hline Norepinephrine $\max \left(\mu \mathrm{g} \bullet \mathrm{kg}^{-1} \bullet \mathrm{min}^{-1}\right)$ & $0.05 \pm 0.03$ & $0.08 \pm 0.04$ & 0.003 \\
\hline Inotropics & $34(76)$ & $23(52)$ & 0.02 \\
\hline Vasopressors within $24 \mathrm{~h}$ postop & $5(11)$ & $11(26)$ & 0.08 \\
\hline Vasopressors within $72 \mathrm{~h}$ postop & $2(4)$ & $5(12)$ & 0.21 \\
\hline Length of hospital stay (days) & $17 \pm 7$ & $19 \pm 9$ & 0.22 \\
\hline
\end{tabular}

Data are presented as number of patients and percentage or mean and standard deviation

GDT goal-directed therapy. Results with a $P$ Value $<0.05$ are considered significant and are written in italic type

hypothesized that EDM GDT leads to a pre-emptive optimization of patients' hemodynamic status, reducing the risk for severe BCIS. Patients of the GDT group experienced an individually tailored preloading with crystalloid fluids according to stroke volume optimization without risking volume overload. Low cardiac output syndrome was avoided by additional use of inotropic therapy also supporting the right ventricle. Apart from that, acute increase of pulmonary vascular resistance as part of BCIS might lead to distension of the right ventricle with a concomitant decrease of coronary blood flow. Therefore keeping up a sufficient systemic blood pressure is crucial [6-8]. This aim was achieved by norepinephrine administration. The results show that although not different at the beginning, EDM GDT led to better cardiac and stroke volume index at the time of cement insertion while mean arterial pressure was comparable between groups. Although cardiac and stroke volume index were significantly higher in the GDT group we were not able to reduce the risk for overall BCIS.

Most studies conducted on intraoperative goal-directed fluid management focused on abdominal surgery using colloids to optimize stroke volume. Inconsistent results were reported [22-27]. In contrast to these studies we did not use colloids to optimize stroke volume due to several known side effects caused by liberal administration. In accordance with current literature we used a combination of goal-directed fluid therapy and inotropic medication instead of single goal-directed fluid therapy [13, 14, 28-30]. Data on EDM-guided goal-directed fluid therapy in orthopedic surgery showed promising results with regards to length of hospital stay $[10,12]$. However, those studies did not focus on patients undergoing cemented hip arthroplasty. In this randomized controlled trial the amount of intraoperative crystalloid fluids were comparable between groups, whereas the GDT group received inotropic medication more frequent but less norepinephrine. Timing of stroke volume optimization with crystalloids seems to be crucial as only $20 \%$ is kept intravascular [31]. The timing of fluid boluses due to different triggers was different between the groups. As a consequence, the overall amount of fluids showed no difference between the groups whereas a different timing led to better cardiac performance in the GDT group. With regards to the difference in catecholamine therapy there was no difference in mean arterial pressure at any time between the groups, although hemodynamic variables differed significantly. This result underlined the fact that norepinephrine primarily acts as a vasopressor leading to an increased afterload, that might in part be responsible for a decreased cardiac performance (cardiac and stroke volume index).

With regards to already known independent predictors of BCIS (ASA $\geq 3$, femoral neck fracture, medication with diuretics and anticoagulation) randomization in this study appears adequate $[5,9,32]$. The high percentage of patients classified as ASA 3 and 4 in this study makes the data presented reliable. The only baseline parameter that differed between groups was the number of patients suffering from chronic kidney disease grade 3 which was significantly higher in the GDT group. Nevertheless, regarding the secondary endpoints the incidence of postoperative acute kidney injury was significantly higher in the control group.

This study has several limitations. A limitation referring to the esophageal Doppler technique to measure stroke volume and cardiac index is the assumption and not individualized measurement of the aortic diameter derived from patients' height and weight. As a result, over- and underestimation of stroke volume can occur due to anatomical anomalies of the descending aorta [19]. Like most of the hemodynamic monitoring devices apart from the pulmonary artery catheter (which is an 
Table $\mathbf{5}$ Intraoperative hemodynamic parameters

\begin{tabular}{|c|c|c|c|c|}
\hline & & $\begin{array}{l}\text { GDT } \\
(n=45)\end{array}$ & $\begin{array}{l}\text { Control } \\
(n=45)\end{array}$ & $P$ Value \\
\hline \multirow[t]{4}{*}{$\mathrm{SD}(\mathrm{cm})$} & pre & $9.9 \pm 3.11$ & $9.5 \pm 2.9$ & 0.49 \\
\hline & before & $10.4 \pm 2.9$ & $9.0 \pm 2.9$ & 0.02 \\
\hline & after & $10.1 \pm 2.9$ & $8.9 \pm 3.1$ & 0.07 \\
\hline & end & $9.6 \pm 3.1$ & $8.6 \pm 3.1$ & 0.12 \\
\hline \multirow[t]{4}{*}{ FTc (ms) } & pre & $293 \pm 42$ & $290 \pm 44$ & 0.69 \\
\hline & before & $303 \pm 37$ & $285 \pm 41$ & 0.03 \\
\hline & after & $298 \pm 44$ & $279 \pm 40$ & 0.04 \\
\hline & end & $289 \pm 49$ & $271 \pm 44$ & 0.07 \\
\hline \multirow[t]{4}{*}{$\mathrm{MA}\left(\mathrm{cm}^{-2} \mathrm{~s}^{-2}\right)$} & pre & $6.4 \pm 2.5$ & $6.3 \pm 2.0$ & 0.77 \\
\hline & before & $6.3 \pm 2.0$ & $6.4 \pm 2.0$ & 0.78 \\
\hline & after & $6.7 \pm 2.0$ & $6.9 \pm 2.5$ & 0.61 \\
\hline & end & $7.1 \pm 2.2$ & $7.5 \pm 2.5$ & 0.52 \\
\hline \multirow[t]{4}{*}{$\mathrm{Cl}\left(\bullet \bullet \mathrm{min}^{-1} \bullet \mathrm{m}^{-2}\right)$} & pre & $2.6 \pm 0.8$ & $2.4 \pm 0.9$ & 0.30 \\
\hline & before & $2.7 \pm 0.7$ & $2.2 \pm 0.7$ & 0.003 \\
\hline & after & $2.8 \pm 0.8$ & $2.4 \pm 1.1$ & 0.04 \\
\hline & end & $2.7 \pm 0.9$ & $2.3 \pm 0.9$ & 0.04 \\
\hline \multirow[t]{4}{*}{ SVI $\left(\mathrm{ml} \bullet \mathrm{m}^{-2}\right)$} & pre & $44 \pm 1$ & $42 \pm 15$ & 0.35 \\
\hline & before & $47 \pm 13$ & $40 \pm 15$ & 0.02 \\
\hline & after & $45 \pm 12$ & $39 \pm 16$ & 0.04 \\
\hline & end & $43 \pm 13$ & $37 \pm 16$ & 0.07 \\
\hline \multirow[t]{4}{*}{$\mathrm{HR}\left(1 \bullet \mathrm{min}^{-1}\right)$} & pre & $59 \pm 14$ & $58 \pm 10$ & 0.60 \\
\hline & before & $61 \pm 13$ & $61 \pm 9$ & 0.99 \\
\hline & after & $64 \pm 14$ & $63 \pm 11$ & 0.54 \\
\hline & end & $66 \pm 15$ & $64 \pm 11$ & 0.39 \\
\hline \multirow[t]{4}{*}{$\mathrm{PV}\left(\mathrm{cm}^{-1} \mathrm{~s}^{-1}\right)$} & pre & $50 \pm 14$ & $49 \pm 14$ & 0.76 \\
\hline & before & $50 \pm 12$ & $48 \pm 15$ & 0.43 \\
\hline & after & $51 \pm 12$ & $49 \pm 16$ & 0.49 \\
\hline & end & $51 \pm 14$ & $50 \pm 16$ & 0.64 \\
\hline \multirow[t]{4}{*}{ BP sys $(\mathrm{mmHg})$} & pre & $124 \pm 16$ & $119 \pm 14$ & 0.12 \\
\hline & before & $129 \pm 15$ & $126 \pm 13$ & 0.24 \\
\hline & after & $134 \pm 16$ & $129 \pm 18$ & 0.15 \\
\hline & end & $122 \pm 14$ & $120 \pm 15$ & 0.43 \\
\hline \multirow[t]{4}{*}{ MAP (mmHg) } & pre & $82 \pm 12$ & $81 \pm 11$ & 0.70 \\
\hline & before & $85 \pm 11$ & $85 \pm 10$ & 0.90 \\
\hline & after & $87 \pm 12$ & $86 \pm 11$ & 0.75 \\
\hline & end & $80 \pm 9$ & $80 \pm 10$ & 0.84 \\
\hline
\end{tabular}

Data are presented as mean and standard deviation

GDT Goal-directed Therapy, MAP mean arterial pressure, HR heart rate, SD stroke distance, FTc corrected flow time, $\mathrm{Cl}$ cardiac index, SVI stroke volume index, $P V$ peak velocity, $B P$ sys systolic blood pressure, $B P$ dia diastolic blood pressure, pre before surgical incision, before before bone cement insertion, after after bone cement insertion, end end of surgery. Results with a $P$ Value $<$ 0.05 are considered significant and are written in italic type

invasive technique with an increased risk for side effects), EDM is not suitable to distinguish between right or left ventricular dysfunction [17]. In agreement with findings of previous studies, postoperative complications, especially the incidence of acute kidney injury was reduced in the GDT compared to the control group [10, 12, 22]. These findings on renal morbidities must be viewed with caution because the study was not sufficiently powered to allow reliable statistical assessment of these outcome variables. Our aim was to detect a reduction of overall BCIS from 28 to $5 \%$, which appears quite optimistic, but due to our a priori clinical experience using this EDM GDT for cemented hip arthroplasties this approach did not appear unrealistic. As a consequence, this study was only powered to detect a difference in the incidence of overall BCIS including grade 1-3 between the GDT and control group. This study is under-powered to draw reliable conclusions regarding the incidence of BCIS grade 2.

\section{Conclusions}

In summary, this is the first randomized controlled trial that focuses on the prevention of BCIS in multimorbid patients who are at increased risk using a semi-invasive technique for a differentiated hemodynamic management. The implementation of EDM GDT in patients undergoing cemented hip arthroplasty was not the reason for the low incidence of BCIS at our department. We were not able to reduce the overall incidence of BCIS by this specific EDM GDT. This trial should be considered as a pilot study for further clinical trials including more medical centers to validate a possible risk reduction for BCIS including more intraoperative variables.

\begin{abstract}
Abbreviations
ACE inhibitor: angiotensin-converting enzyme inhibitor; ARBs: Angiotensinreceptor-II blockers; ASA: American Society of Anesthesiologists; BCIS: Bone cement implantation syndrome; BP dia: diastolic blood pressure; BP sys: systolic blood pressure; CAD: coronary artery disease; Cl: Cardiac index; EDM: Esophageal Doppler monitoring; FTc: corrected flow time; GDT: goaldirected therapy; GFR: glomerular filtration ratio; HR: heart rate; MAP: Mean arterial pressure; PV: peak velocity; SD: stroke distance; SVI: stroke volume index
\end{abstract}

Funding

This work was supported by departmental funding.

Availability of data and materials

The datasets generated and analyzed during the current study are available from the corresponding author on reasonable request.

Authors' contributions

Study design: KBK, WB, TL, UG Protocol design: KBK, WB, SH, UG Advisor for study protocol and management of the study: KBK, SH, HB, UG Patient recruitment: KBK, WB, JR, LK Data collection: KBK, JR, WB, LK, TL, UG Study conduct: KBK, JR, SH, TL, UG Study monitoring: KBK, WB, TL, SH, LK, HB, UG Data analysis: KBK, JR, WB, SH, UG Data evaluation: KBK, TL, HB, UG Writing the manuscript: KBK, SH, UG Editing and approval of the manuscript: KBK, WB, JR, LK, SH, TL, HB, UG.

\section{Ethics approval and consent to participate}

This randomized clinical two-arm parallel study was approved by the local Ethics Committee, Freiburg, Germany [EK 160/15, PI: U. Goebel] and registered in the German Clinical Trials Register (DRKS No. 00008778, 16th of June, 2015). Written informed consent to participate in this study was obtained from each patient prior to study enrollment. 


\section{Consent for publication}

Consent for publication was obtained from each patient enrolled in this study.

\section{Competing interests}

The authors declare that they have no competing interests.

\section{Publisher's Note}

Springer Nature remains neutral with regard to jurisdictional claims in published maps and institutional affiliations.

\section{Author details}

'Department of Anaesthesiology and Critical Care, Medical Center University of Freiburg, Hugstetter Strasse 55, 79106 Freiburg, Germany. ${ }^{2}$ Department of Orthopaedic and Trauma Surgery, Medical Center University of Freiburg, Freiburg, Germany. ${ }^{3}$ Faculty of Medicine, University of Freiburg, Freiburg, Germany.

Received: 23 January 2018 Accepted: 25 May 2018

Published online: 06 June 2018

\section{References}

1. Learmonth ID, Young C, Rorabeck C. The operation of the century: total hip replacement. Lancet. 2007:370:1508-19.

2. Kurtz S, Ong K, Lau E, Mowat F, Halpern M. Projections of primary and revision hip and knee arthroplasty in the United States from 2005 to 2030. J Bone Joint Surg Am. 2007;89:780-5.

3. Smith AJ, Dieppe P, Vernon K, Porter M, Blom AW. National Joint Registry of England and Wales. Failure rates of stemmed metal-on-metal hip replacements: analysis of data from the National Joint Registry of England and Wales. Lancet. 2012:379:1199-204.

4. Griffiths R, White SM, Moppett IK, Parker MJ, Chesser TJS, Costa ML, et al. Safety guideline: reducing the risk from cemented hemiarthroplasty for hip fracture 2015. Anaesthesia. 2015;70:623-6.

5. Olsen F, Kotyra M, Houltz E, Ricksten S-E. Bone cement implantation syndrome in cemented hemiarthroplasty for femoral neck fracture: incidence, risk factors, and effect on outcome. Br J Anaesth. 2014;113:800-6.

6. Donaldson AJ, Thomson HE, Harper NJ, Kenny NW. Bone cement implantation syndrome. Br J Anaesth. 2009;102:12-22.

7. Kotyra M, Houltz E, Ricksten S-E. Pulmonary haemodynamics and right ventricular function during cemented hemiarthroplasty for femoral neck fracture. Acta Anaesthesiol Scand. 2010:54:1210-6.

8. Urban MK, Sheppard R, Gordon MA, Urquhart BL. Right ventricular function during revision total hip arthroplasty. Anesth Analg. 1996;82:1225-9.

9. Hamal PK, Poudel PR, Singh J. Grade III bone cement implantation syndrome in malignant lung cancer patient: a case report. BMC Anesthesiol. 2018;18:28.

10. Sinclair S, James $S$, Singer $M$. Intraoperative intravascular volume optimisation and length of hospital stay after repair of proximal femoral fracture: randomised controlled trial. BMJ. 1997;315:909-12.

11. Cecconi M, Fasano N, Langiano N, Divella M, Costa MG, Rhodes A, et al. Goal-directed haemodynamic therapy during elective total hip arthroplasty under regional anaesthesia. Crit Care. 2011;15:R132.

12. Venn $R$, Steele A, Richardson P, Poloniecki J, Grounds M, Newman P. Randomized controlled trial to investigate influence of the fluid challenge on duration of hospital stay and perioperative morbidity in patients with hip fractures. Br J Anaesth. 2002;88:65-71.

13. Feldheiser A, Conroy P, Bonomo T, Cox B, Garces TR, Spies C, et al. Development and feasibility study of an algorithm for intraoperative goaldirected Haemodynamic Management in Noncardiac Surgery. J Int Med Res. 2012;40:1227-41.

14. Kaufmann KB, Stein L, Bogatyreva L, Ulbrich F, Kaifi JT, Hauschke D, et al. Oesophageal Doppler guided goal-directed haemodynamic therapy in thoracic surgery - a single Centre randomized parallel-arm trial. $\mathrm{Br} \mathrm{J}$ Anaesth. 2017;118:852-61.

15. Giglio MT, Marucci M, Testini M, Brienza N. Goal-directed haemodynamic therapy and gastrointestinal complications in major surgery: a meta-analysis of randomized controlled trials. Br J Anaesth. 2009;103:637-46.

16. Downs EA, Isbell JM. Impact of hemodynamic monitoring on clinical outcomes. Best Pract Res Clin Anaesthesiol. 2014;28:463-76.
17. Sandham JD, Hull RD, Brant RF. A randomized, controlled trial of the use of pulmonary-artery catheters in high-risk surgical patients. NEngl J Med. 2003; 348(1):5-14

18. Moriyama M, Watanabe S, Hiraki T, Kano T, Okawa T, Ishibashi M. Relationship between intraoperative transoesophageal echocardiography findings and perfusion lung scintigraphy results on first postoperative day. Br J Anaesth. 2005;94:607-12.

19. Cholley BP, Singer M. Esophageal Doppler: noninvasive cardiac output monitor. Echocardiography. 2003;20:763-9.

20. Jammer I, Wickboldt N, Sander M, Smith A, Schultz MJ, Pelosi P, et al. Standards for definitions and use of outcome measures for clinical effectiveness research in perioperative medicine: European perioperative clinical outcome (EPCO) definitions: a statement from the ESA-ESICM joint taskforce on perioperative outcome measures. Eur J Anaesthesiol. 2015:88-105.

21. Koessler MJ, Fabiani R, Hamer H, Pitto RP. The clinical relevance of embolic events detected by transesophageal echocardiography during cemented total hip arthroplasty: a randomized clinical trial. Anesth Analg. 2001;92:49-55.

22. Gan TJ, Soppitt A, Maroof M, el-Moalem H, Robertson KM, Moretti E, et al. Goal-directed intraoperative fluid administration reduces length of hospital stay after major surgery. Anesth. 2002;97:820-6.

23. Conway DH, Mayall R, Abdul-Latif MS, Gilligan S, Tackaberry C. Randomised controlled trial investigating the influence of intravenous fluid titration using oesophageal Doppler monitoring during bowel surgery. Anaesthesia. 2002:57:845-9.

24. Srinivasa S, Kahokehr A, Soop M, Taylor M, Hill AG. Goal-directed fluid therapy- a survey of anaesthetists in the UK, USA, Australia and New Zealand. BMC Anesthesiol. 2013;13:5.

25. Phan TD, D'Souza B, Rattray MJ, Johnston MJ, Cowie BS. A randomised controlled trial of fluid restriction compared to oesophageal Dopplerguided goal-directed fluid therapy in elective major colorectal surgery within an enhanced recovery after surgery program. Anaesth Intensive Care. 2014;42:752-60.

26. Pestaña D, Espinosa E, Eden A, Nájera D, Collar L, Aldecoa C, et al. Perioperative goal-directed hemodynamic optimization using noninvasive cardiac output monitoring in major abdominal surgery: a prospective, randomized, multicenter, pragmatic trial: POEMAS study (PeriOperative goal-directed thErapy in major abdominal surgery). Anesth Analg. 2014;119:579-87.

27. Gómez-Izquierdo JC, Trainito A, Mirzakandov D, Stein BL, Liberman S, Charlebois $\mathrm{P}$, et al. Goal-directed fluid therapy does not reduce primary postoperative ileus after elective laparoscopic colorectal surgery: a randomized controlled trial. Anesthesiology. 2017;127:36-49.

28. Perner A, Haase N, Guttormsen AB, Tenhunen J, Klemenzson G, Åneman A, et al. Hydroxyethyl starch 130/0.42 versus Ringer's acetate in severe sepsis. N. Engl. J. Med, 34. 2012;367:124

29. Grocott MPW, Dushianthan A, Hamilton MA, Mythen MG, Harrison D, Rowan $\mathrm{K}$, et al. Perioperative increase in global blood flow to explicit defined goals and outcomes after surgery: a Cochrane systematic review. Br J Anaesth. 2013;111:535-48.

30. Hamilton MA, Cecconi M, Rhodes A. A systematic review and meta-analysis on the use of preemptive hemodynamic intervention to improve postoperative outcomes in moderate and high-risk surgical patients. Anesth Analg. 2011;112:1392-402.

31. Yates DRA, Davies SJ, Milner HE, Wilson RJT. Crystalloid or colloid for goaldirected fluid therapy in colorectal surgery. Br J Anaesth. 2014;112:281-9.

32. Pietak S, Holmes J, Matthews R, Petrasek A, Porter B. Cardiovascular collapse after femoral prosthesis surgery for acute hip fracture. Can J Anaesth. 1997; 44:198-201. 